\title{
ON THE UNIQUENESS OF GENERALIZED MATRIX FUNCTIONS
}

\author{
L. B. BEASLEY AND L. J. CUMMINGS
}

\begin{abstract}
We consider the sets of "singular" matrices corresponding to generalized matrix functions. The latter are generalizations of the determinant function. It is shown that two generalized matrix functions determine the same set of "singular" matrices if and only if they differ by a scalar multiple.
\end{abstract}

Let $M_{n}$ denote the set of all $n \times n$ matrices over a field $F$ and let $S_{n}$ be the group of all permutations of $\{1, \ldots, n\}$. We denote the identity of $M_{n}$ by $I$ and the identity of $S_{n}$ by $e$. For each mapping $\chi: S_{n} \rightarrow F$ we define the generalized matrix function $d_{\chi}: M_{n} \rightarrow F$ by

$$
d_{\chi}(A)=\sum_{\sigma \in S_{n}} \chi(\sigma) a_{1 \sigma(1)} \cdots a_{n \sigma(n)}
$$

where $A=\left(a_{i j}\right) \in M_{n}$. These functions have been studied extensively when $\chi$ is the trivial extension of an irreducible character of a subgroup of $S_{n}$.

In particular, if $\chi$ is the alternating character, $\operatorname{sgn}(\sigma)= \pm 1$, depending on the parity of $\sigma \in S_{n}$, then $d_{\chi}$ is the determinant function; or, if $\chi \equiv 1$ then $d_{\chi}$ is the permanent function. Recent interest in the topic of generalized matrix functions was initiated by Marcus and Minc [1] although they appear to have been first introduced by Schur [4].

The singular matrices in $M_{n}$ are precisely those on which the determinant function vanishes. We study here the relationship between the generalized matrix functions and the corresponding sets of "singular" matrices. We define the singular set of the generalized matrix function $d_{\chi}$ by

$$
Z(\chi)=\left\{A \in M_{n} \mid d_{\chi}(A)=0\right\}
$$

and prove that $d_{\chi}$ is essentially determined by $Z(\chi)$. In particular, $Z(\operatorname{sgn})$ is the set of classical singular matrices and clearly $Z(0)=M_{n}$.

Recently R. Merris [3] has shown that if $\chi$ is an irreducible complex character of some subgroup of $S_{n}$ then the determinant is the only generalized matrix function which is nonzero on every matrix in $Z(\operatorname{sgn})$. Equivalently, for such a character $Z(\chi) \subseteq Z\left(\right.$ sgn) implies $d_{\chi}=$ det. The same conclusion is obtained by Marcus [2, p.

Received by the editors February 2, 1982 and, in revised form, May 25, 1982.

1980 Mathematics Subject Classification. Primary 15A15.

Key words and phrases. Generalized matrix functions, singular matrices.

'The work of the first author was supported by the National Science Foundation Grant MCS 7904472. The work of the second author was supported by the Natural Sciences and Engineering Research Council Canada grant AS285. 
151] but with the hypothesis $Z(\operatorname{sgn}) \subseteq Z(\chi)$. Both of these results are easy consequences of the theorem proved below. Marcus' proof suggests that a more general result could be obtained by use of the Hilbert Nullstellensatz [5, pp. 164-167]. However, we obtain a more general result by a direct argument.

For $\psi: S_{n} \rightarrow F$ and $\alpha \in S_{n}$ we define

$$
\psi_{\eta}(\alpha)=\psi(\alpha \circ \eta)
$$

for each $\eta \in S_{n}$. The support of $\chi: S_{n} \rightarrow F$ is

$$
s(\chi)=\left\{\alpha \in S_{n} \mid \chi(\alpha) \neq 0\right\} .
$$

The following lemma lists some readily verified facts which we will use in the sequel without explicit mention.

Lemma. Let $\psi, \chi: S_{n} \rightarrow F$ be arbitrary mappings and $\eta \in S_{n}$, then

(i) $s(\chi)=s(\psi)$ if and only if $s\left(\chi_{\eta}\right)=s\left(\psi_{\eta}\right)$,

(ii) $a \chi=b \psi$ if and only if $a \chi_{\eta}=b \psi_{\eta} ; a, b \in F$,

(iii) $P_{\eta}=\left(\delta_{i \eta(j)}\right)$ implies $d_{\chi_{\eta}}(A)=d_{\chi}\left(P_{\eta}^{\prime} A\right), A \in M_{n}$;

(iv) $Z(\chi) \subseteq Z(\psi)$ if and only if $Z\left(\chi_{\eta}\right) \subseteq Z\left(\psi_{\eta}\right)$.

THEOREM. Let $\chi, \psi: S_{n} \rightarrow F$ be any two functions where $F$ is an arbitrary field. Then $Z(\chi) \subset Z(\psi)$ if and only if $\psi=a \chi$ for some $a \in F$. Further, if $a \neq 0$ then $Z(\chi)=$ $Z(\psi)$.

Proof. Assume $Z(\chi) \subset Z(\psi)$. If $s(\psi)$ is empty then $Z(\psi)=M_{n}$ and the implication follows with $a=0$. If $e \notin s(\psi)$ choose $\eta$ in (2) so that $e \in s\left(\psi_{\eta}\right)$. Thus we assume $e \in s(\psi)$, further, $e \in s(\chi)$. Otherwise, $d_{\chi}(I)=0$ and $I \in Z(\chi) \subseteq Z(\psi)$ would imply the contradiction $0=d_{\psi}(I)=\psi(e)$. Therefore, there exists $a \in F$ such that $\psi(e)=a \chi(e)$.

If it is false that

$$
\psi(\tau)=a \chi(\tau)
$$

for all $\tau \in S_{n}$ then

$$
R=\left\{\tau \in S_{n} \mid \psi(\tau) \neq a \chi(\tau)\right\}
$$

is nonempty and does not contain $e$. Choose a $\sigma \in R$ with the fewest number of cycles of length greater than 1 .

Let $T$ be the set of disjoint cycles whose product is $\sigma$. Since $\sigma \neq e, T$ contains a nontrivial cycle. Let $L$ denote a maximal subset of $T$ such that the product of cycles in $L$ lies in $s(\chi)$. Since $e \in s(\chi), L$ is nonempty. Let $\rho$ be the product of all cycles in $L$ and note that $\rho \neq \sigma$.

There is a permutation matrix $S$ and integer $k$ such that

$$
S P_{\sigma} S^{t}=\left[\begin{array}{cc}
P_{1} & 0 \\
0 & P_{2}
\end{array}\right] \text { and } S P_{\rho} S^{t}=\left[\begin{array}{cc}
I_{k} & 0 \\
0 & P_{2}
\end{array}\right]
$$

where $P_{1}$ has all 0 's on the main diagonal and $P_{2}$ may be empty. Let

$$
E_{1}=\left[\begin{array}{cc}
\chi(\rho) & 0 \\
0 & I_{n-1}
\end{array}\right], \quad E_{2}=\left[\begin{array}{cc}
-\chi(\sigma) & 0 \\
0 & I_{n-1}
\end{array}\right]
$$


and

$$
A=E_{1} S P_{\sigma} S^{t}+E_{2} S P_{p} S^{t}
$$

Set

$$
m=\#\{i \mid \sigma(i)=\rho(i)\},
$$

which is the number of rows in $P_{2}$ above. Directly from definition (1) we determine that

$$
d_{\chi}\left(S^{\prime} A S\right)=2^{m}[\chi(\sigma) \chi(\rho)-\chi(\rho) \chi(\sigma)]=0 .
$$

For, if both $a_{1 \tau(1)} \cdots a_{n \tau(n)}$ and $\chi(\tau)$ are nonzero then $\tau=\sigma$ or $\tau=\rho$, by the choice of $\sigma$ and the definition of $\rho$. Since $Z(\chi) \subseteq Z(\psi)$ we have $d_{\psi}\left(S^{t} A S\right)=0$ as well and

$$
a d_{\psi}\left(S^{t} A S\right)=a \chi(\rho) \psi(\sigma)-a \chi(\sigma) \psi(\rho)=0 .
$$

Since $\rho$ has fewer cycles that $\sigma$,

$$
\psi(\rho)=a \chi(\rho)
$$

Therefore, (4) becomes

$$
a d_{\psi}\left(S^{t} A S\right)=\psi(\rho)[\psi(\sigma)-a \chi(\sigma)] .
$$

Also $\psi(\rho)$ is nonzero since $s(\chi) \subseteq s(\psi)$ and $\rho \in s(\chi)$. Since $F$ is a field we conclude

$$
\psi(\sigma)=a \chi(\sigma)
$$

contradicting the choice of $\boldsymbol{\sigma}$.

For the converse, assume $\psi=a \chi$. Then for $A \in M_{n}$,

$$
\begin{aligned}
\operatorname{ad}_{\chi}(A) & =\sum_{\tau \in S_{n}} a \chi(\tau) \prod_{i=1}^{n} a_{i \tau(i)} \\
& =\sum_{\tau \in S_{n}} \psi(\tau) \prod_{i=1}^{n} a_{i \tau(i)}=d_{\psi}(A) .
\end{aligned}
$$

If $A \in Z(\chi)$ then $0=a d_{\chi}(A)=d_{\psi}(A)$, and $A \in Z(\psi)$.

The following corollaries contain some immediate but interesting consequences of the above theorem.

COROLlaRY 1. If $\chi, \psi: S_{n} \rightarrow F$ are any functions and $Z(\chi) \underset{\neq}{\subset} Z(\psi)$ then $\psi=0$ and $Z(\psi)=M_{n}$.

COROllary 2. If $\chi: S_{n} \rightarrow F$ is any function then $d_{\chi}(A)=0$ for all noninvertible matrices $A$ if and only if $d_{\chi}=\operatorname{det}$.

COROllaRY 3. If $\chi: S_{n} \rightarrow F$ is any function then $d_{\chi}(A) \neq 0$ for all invertible $A$ if and only if $d_{\chi}=$ det.

The results of Marcus and Merris mentioned previously are special cases of the last two corollaries. 


\section{REFERENCES}

1. M. Marcus and H. Minc, Generalized matrix functions, Trans. Amer. Math. Soc. 116 (1965), 316-329.

2. M. Marcus, Finite dimensional multilinear algebra, Dekker, New York, 1973.

3. R. Merris, Generalized matrix functions: a research problem, Linear and Multilinear Algebra 8 (1979), 83-86.

4. I. Schur, Ueber endiche Gruppen und Hermitesche Formen, Math. Z. 1 (1918), 184-207.

5. O. Zariski and P. Samuel, Commutative algebra, Vol. II, Van Nostrand, New York, 1960.

Department of Mathematics, Utah State University, Logan, Utah 84322

Department of Mathematics, University of Waterloo, Waterloo, Ontario, Canada N2L 3Gl 